\title{
Medicamentos Genéricos: Uma Alternativa para o Mercado Brasileiro
}

\author{
Generic Drugs: An Alternative for the Brazilian Market \\ Jorge Bermudez ${ }^{1}$

\begin{abstract}
BERMUDEZ, F. Generic Drugs: An Alternative for the Brazilian Market. Cad. Saúde Públ., Rio de Faneiro, 10 (3): 368-378, ful/Sep, 1994.
\end{abstract}

The analysis of the main characteristics of the pharmaceutical industry worldwide and market aspects of that industry in Brazil leads us to consider a high share of external dependency and oligopolization.

This situation is not peculiar to our country, but is rather a global trend.

Recent Government initiatives in Brazil, aiming at reducing that dependency, associated with the critical aspects regarding the prices and costs of drugs as compared to international prices, are the background that support the proposal of evaluating generic drugs as an alternative for Brazilian market. One of the most striking aspect is the verification of overpricing of raw materials as demonstrated by "transfer prices", a practice that has been observed in several countries.

Analysing international concepts dealing with generic drugs, generic names and pharmaceutical equivalence, based on World Health Organization recomendations and evaluating the experiences of different countries, several actions are proposed in order to gradually enforce a policy of generic drugs, bioequivalent and interchangeable, as a possible regulatory mechanisms for Brazil.

Key words: Generic Drugs; Drug Policies and Health; Drug Market; Pharmaceutical Industry

\section{INTRODUÇÃO}

\section{As Características Mercadológicas da Indústria Farmacêutica no Brasil}

A discussão referente aos medicamentos genéricos, sua inserção mundial e sua avaliação enquanto uma alternativa para o mercado brasileiro, se encontra associada a uma reflexão a respeito do nosso modelo de atenção à saúde, além das características mercadológicas da indústria farmacêutica no Brasil e no mundo. Essa discussão tem que levar em consideração a própria conceituação de medicamentos enquanto insumos em saúde ou como mercadorias que objetivam exclusivamente lucro.

O mercado mundial de medicamentos nas economias capitalistas está estimado em 170

${ }_{1}^{1}$ Departamento de Ciências Biológicas da Escola Nacional de Saúde Pública. Rua Leopoldo Bulhões, 1480, $6^{\circ}$ andar, Rio de Faneiro, RF, 21041-210, Brasil. bilhões de dólares anuais (Codetec, 1991a; Scrip, 1990). O Brasil se situa como o nono mercado mundial, com um faturamento que hoje ultrapassa os três bilhões de dólares. Entretanto, podemos verificar que o consumo per capita é muito baixo, quando comparado com outros países (mais de dez vezes menos do que os EUA e países da Comunidade Européia).

Como características globais da indústria farmacêutica, podemos destacar a internacionalização, a diversificação e a concentração (Bermudez, 1994).

De uma maneira geral, dois aspectos devem ser ressaltados ao analisar a situação da indústria farmacêutica no Brasil: a dependência e a oligopolização, refletindo uma tendência mundial. Estes dois componentes ficam evidentes em ambos setores que compõem a indústria farmacêutica no seu sentido mais global: a Química Fina e a Formulação Farmacêutica.

O setor de Química Fina abrange a elaboração de produtos químicos de maior valor 
agregado que os da indústria química de base, classificados como intermediários ou especialidades. Entre as principais características dos intermediários, destacamos sua compe-titividade dependente da tecnologia de produção, vulnerabilidade ao progresso tecnológico, proteção à propriedade industrial com foco no patenteamento dos processos produtivos, comercialização por especificações e um mercado concentrado, com poucos compradores e poucos produtores. Já o segmento das especialidades se caracteriza por apresentar competitividade dependente do desempenho do produto, importância da tecnologia de aplicação dos produtos, proteção industrial enfatizando o patenteamento dos produtos, comercialização com assistência técnica e um grande número de empresas atuando no mercado. $\mathrm{O}$ mercado mundial do complexo da química fina é estimado em US $\$ 256$ bilhões, com 200 bilhões atribuídos a especialidades. O Brasil representa cerca de $3,2 \%$ do mercado mundial. O parque industrial brasileiro do complexo de química fina possui uma capacidade instalada mensurável de $500 \mathrm{mil}$ toneladas anuais. A situação que se observa nas empresas nacionais é a utilização de tecnologias desenvolvidas no exterior, enquanto que as empresas multinacionais importam de suas matrizes a tecnologia aplicada nos seus processos de produção e vendas (Abifina/ ABQ/ABEQ, 1992). De acordo com levantamento procedido pela Codetec (1991b), as maté-rias-primas produzidas internamente por empresas nacionais representam menos de $10 \%$ do valor total da produção.

Nossa dependência externa é nítida e pode ser analisada associada à questão da oligopolização do mercado. Uma diferença marcante entre as grandes empresas multinacionais e nossas indústrias brasileiras é que as primeiras procuram verticalizar sua produção, atuando em todos os estágios tecnológicos, incluindo a pesquisa, desenvolvimento de matérias-primas, formulação e marketing (Frenkel et al., 1978), enquanto que as empresas nacionais em sua maioria desenvolvem apenas a etapa de formulação farmacêutica, mantendo a dependência na aquisição das matérias-primas.

A indústria de formulação farmacêutica no Brasil é representada por um setor que movimenta anualmente mais de três bilhões de dólares (Codetec, 1991a; 1991b), sendo um dos dez maiores mercados do mundo. Com um consumo per capita baixo, apresenta uma distribuição perversa, considerando que $23 \%$ da população consomem $60 \%$ da produção (Codetec, 1991a). O mercado governamental representa em torno de $35 \%$ do mercado total. Nossa dependência fica evidente quando constatamos que as empresas multinacionais são responsáveis por 75 a $85 \%$ do faturamento anual no Brasil (Alanac, 1989; Codetec, 1991a; Ceme, 1987a; 1987b). A situação se configura de gravidade quando se constata, a par de um processo crescente de desnacionalização do setor privado, uma ação governamental que não fortalece os laboratórios estatais de produção.

\section{AS INICIATIVAS GOVERNAMENTAIS NO BRASIL: O SONHO DA FARMOBRÁS, O CDI E A CEME}

Cabe lembrar as iniciativas governamentais brasileiras no que se refere ao desenvolvimento recente da indústria farmacêutica nacional e, principalmente, as tentativas buscando a diminuição de nossa dependência externa. Nesse sentido, inicialmente referimos um projeto que começou a ser discutido há pelo menos 30 anos e que não chegou a ser implementado, de estruturar uma sociedade por ações, a Farmoquímica Brasileira S/A. (Farmobrás), tendo como objetivo o estabelecimento de um centro de pesquisas para a produção nacional de matérias-primas. Ao mesmo tempo, se discutia a proposta de estabelecer o monopólio da União na importação de matérias-primas para a indústria farmacêutica brasileira, inclusive tendo sido apresentados projetos de lei. Na mesma proposta, se propunha a constituição de Conselho Nacional da Indústria Farmacêutica, diretamente vinculado à Presidência da República (Palácios, 1962).

Das iniciativas governamentais que efetivamente foram implementadas e que se constituem em marcos da política de medicamentos no país, podemos caracterizar duas vertentes diferenciadas. Por um lado, temos uma vertente mais vinculada à política industrial, que é a criação do Conselho de Desenvolvimento Industrial (CDI), a partir de 1965, extinto em 1990. Como antecedentes, podemos citar a 
Comissão Parlamentar de Inquérito da indústria farmacêutica iniciada em 1961, a nomeação de Grupo de Trabalho em 1963 e a constituição do Grupo Executivo da Indústria Farmacêutica (Geifar), cujas atividades passaram a ser absorvidas pelo Conselho e seus grupos executivos.

A outra vertente, mais vinculada, não no seu início, mas com a evolução histórica, às ações de saúde, foi a criação da Central de Medicamentos (Ceme), em 1971. Discutimos o seu desempenho em trabalhos anteriores (Bermudez, 1992; 1994), as pressões e as distorções que levaram a que, ultimamente, suas atividades se restringissem à aquisição centralizada de medicamentos, insuficiente para o atendimento da demanda da rede de serviços de saúde. Todas as idéias autonomistas, de investimento em pesquisa e desenvolvimento, de subsistemas interagindo na política de medicamentos no país, presentes nas propostas que fundamentaram sua criação, foram se perdendo ao longo das mudanças de vinculação, das restrições orçamentárias e da lamentável história mais recente que chegou a vincular as atividades da Ceme com esquemas de corrupção denunciados no Governo Federal.

Em que pese as diversas iniciativas de implementação de ações concretas na política de medicamentos no Brasil, os indicadores econômicos revelam o pouco investimento que está sendo realizado em saúde e em pesquisa e desenvolvimento, podendo agravar o quadro concentrador. Gerez (1993b) avalia que a participação dos países em desenvolvimento no mercado farmacêutico mundial vem caindo de maneira considerável. $\mathrm{O}$ percentual de gastos públicos com saúde na maioria desses países oscila em torno de 1 a $2 \%$ do Produto Interno Bruto (PIB), enquanto que nos países industrializados esse percentual é muito maior. Os dados do Banco Mundial (1993) mostram que em 1990, o Brasil investiu 4,2\% do PIB em saúde, enquanto que os Estados Unidos investiram 12,7\%. Da mesma maneira, os dispêndios com pesquisa e desenvolvimento em vários países mostram uma relação direta entre esses gastos e a capacidade de inovação tecnológica. De acordo com Martinez \& Gonçalves (1990), enquanto que os Estados Unidos investem 2,94\% do seu PIB em pesquisa e desenvolvimento, este investimento no Brasil se situa em apenas $0,4 \%$.

\section{A CODETEC E INICIATIVAS EMPRESARIAIS}

Ao analisarmos as iniciativas governamentais, temos que levar em consideração também a implantação da Companhia de Desenvolvimento Tecnológico (Codetec), criada em 1976 como empresa privada, mas inserida em prioridades estabelecidas pelo governo (Codetec, 1991c). A partir de 1984, com base na decisão conjunta da Secretaria de Tecnologia Industrial, Ceme e Conselho Nacional de Desenvolvimento Científico e Tecnológico (CNPq), a Codetec passou a priorizar o desenvolvimento de processos na área de síntese química de fármacos. No passado mais recente, a falta de repasse financeiro por parte da Ceme quase que levou à falência desta empresa. De qualquer maneira, tem que ser considerada como um modelo no que se refere à possibilidade do país contar com centros de excelência voltados para a capacitação nacional, a incorporação de tecnologias e a diminuição da nossa dependência externa.

Não podemos deixar de mencionar também as atividades desenvolvidas pelo Instituto de Tecnologia em Fármacos (Far-Manguinhos/ Fiocruz), em conjunto com a empresa Nordeste Tecnologia (Nortec/Norquisa) na síntese de fármacos que hoje são produzidos em escala industrial no país.

Como iniciativas empresariais de importância, considerando que incorporaram tecnologia de produção que substituíram a importação de matérias-primas, cabe mencionar a Bioquímica do Brasil (Biobrás), Companhia Brasileira de Antibióticos (Cibran), Microbiológica e Nortec/ Norquisa. Consideramos que uma política de medicamentos em um país com as características do Brasil tem que considerar e promover mecanismos de consolidação e incentivo a empreendimentos desse tipo.

\section{A QUESTÃO DOS PRECOS E CUSTOS, REAJUSTES, PREÇOS INTERNACIONAIS E FATURAMENTO}

Um último aspecto a considerar é a questão dos preços e custos dos produtos farmacêuticos. O enfrentamento do Ministério da Saúde com a indústria farmacêutica em 1993, que a imprensa 
convencionou chamar de "guerra dos remédios", teve seu início deflagrado pelos reajustes que a indústria vinha impondo nos preços de venda dos medicamentos de uso contínuo.

A Associação Brasileira da Indústria Farmacêutica (Abifarma) defende que o preço médio dos medicamentos no Brasil é dos mais baixos do mundo (Abifarma, 1987). Entretanto, o "preço médio" representa um indicador inadequado que não leva em consideração a essencialidade dos medicamentos, seu consumo, duração do tratamento e outros fatores. Por outro lado, não está também levando em consideração o custo de vida nem o salário mínimo vigente nos diferentes países, que condicionam o poder aquisitivo da população.

Outro aspecto a considerar é que, sistematicamente, os reajustes de preços de medicamentos no Brasil, desde que se desregulamentou o mercado, têm sido superiores à inflação
(Abbas \& Bermudez, 1993). Comparando os preços no país com os preços internacionais, verificamos diferenças difíceis de justificar, sendo que alguns produtos são vendidos aqui a preços até 20 vezes mais caros do que no mercado mundial (Tabela 1).

Na realidade, o mercado brasileiro é altamente dependente de empresas multinacionais, hegemônicas nas vendas. Um fator que precisa ser aprofundado e corrigido pelo governo é a questão do superfaturamento das matérias-primas que entram na composição do custo dos medicamentos nas transações matriz-filial das empresas multinacionais. A Tabela 2 mostra nitidamente que, através dos denominados preços de transferência, as filiais de multinacionais importam matérias-primas de suas matrizes com preços muito superiores aos praticados no mercado mundial (Alifar, 1988; Colômbia, 1992; Gerez, 1993a).

TABELA 1. Preços de Alguns Medicamentos no Brasil e no Mercado Internacional (US\$/Unidade)

\begin{tabular}{lcc}
\hline \hline Medicamento & Brasil $^{\star}$ & Unicef $^{* *}$ \\
\hline Acetaminofen & 0,17 & 0,01 \\
Sulfametoxazol e Trimetoprima & 0,21 & 0,01 \\
Amoxicilina 500 mg & 0,54 & 0,08 \\
Furosemida & 0,09 & 0,005 \\
Ácido Acetilsalicílico & 0,02 & 0,008 \\
Metildopa 250 mg & 0,14 & 0,03 \\
Hidróxido de Alumínio & 0,04 & 0,007 \\
Fenobarbital 100 mg & 0,02 & 0,01 \\
\hline \hline & &
\end{tabular}

TABELA 2. Preços de Transferência: Superfaturamento de Matérias-Primas na Importação

\begin{tabular}{lrrr}
\hline \hline Substância & Preço Multinacional & Preço Internacional \\
\hline Betametasona & 47,50 & 5,00 & $*$ \\
Diazepam & $2.360,00$ & 36,00 & $*$ \\
Gentamicina & $8.000,00$ & 200,00 & $*$ \\
Piroxicam & $12.076,00$ & 150,00 & $*$ \\
& $8.930,00$ & 400,00 & $* *$ \\
Nifedipina & $2.970,00$ & 143,00 & $* *$ \\
Minoxidil & $7.000,00$ & 900,00 & $* *$ \\
Praziquantel & $1.160,00$ & 450,00 & $* * *$ \\
Indometacina & 250,00 & 53,00 & $* * *$ \\
\hline \hline
\end{tabular}

Preços em US\$/Kilo / Fontes: * Asociación Latinoamericana de Industrias Farmacéuticas (Alifar, 1988). (Chile); ** Los Medicamentos en Colombia, 1992. (Colômbia, 1992). (Colômbia) /*** Gerez, J. C. C., 1993a; (Brasil) 


\section{POR QUE OS MEDICAMENTOS GENERICOS? CONCEITOS, EQUIVALÊNCIADEMEDICAMENTOS}

Com o objetivo de discutir especificamente a questão dos medicamentos genéricos, inicialmente é necessário deixar claros uma série de conceitos.

Quanto à denominação ou nome dos medicamentos, podemos diferenciar como segue (Korolkovas, s/d):

a. Sigla, número ou designação de código esta denominação não identifica a natureza do produto e é de caráter provisório. Alguns nomes costumam continuar a ser utilizados (AZT, abreviatura de azidotimidina, cujo nome genérico é zidovudina).

b. Nome químico, que descreve a estrutura química. É necessário esclarecer que o nome químico não corresponde ao nome genérico.

c. Nome registrado é o nome de marca ou comercial ou de fantasia, que confere um verdadeiro monopólio da marca às empresas.

d. Nome genérico: é o nome comum pelo qual é conhecida a substância, escolhido pelos órgãos oficiais. Não é o nome químico. Normalmente o nome genérico se baseia nas Denominações Comuns Internacionais (DCI). No Brasil, a Portaria 971 do Ministério da Saúde, de 10/8/93, atualizou as Denominações Comuns Brasileiras, com base em revisã procedida pela Comissão Permanente de Revisão da Farmacopéia Brasileira.

e. Sinônimos são nomes dados pelos fabricantes ou nomes antigos que continuam sendo usa dos, que não correspondem às DCI. O exemplo mais claro é a dipirona ou dipirona sódica, denominação aceita no Brasil para o metamizol sódico (nome genérico internacional).

Ficando claros os distintos nomes que um produto farmacêutico pode ter, é necessário também discutir o que diferencia determinados produtos com características específicas (WHO, 1993b):

a. Medicamento Genérico - as mais recentes recomendações da Organização
Mundial da Saúde (OMS) tentam evitar o uso do termo "medicamentos genéricos", optando por usar "produto farmacêutico intercambiável". De qualquer maneira, entende-se como conceito de medicamento genérico um produto farmacêutico, que pretende ser intercambiável com o produto inovador, geralmente produzido sem licença da companhia inovadora e comercializado após a expiração da proteção patentária ou outros direitos de exclusividade. Os medicamentos genéricos podem ser comercializados sob a denominação genérica ou utilizar uma nova marca, podendo também apresentar dosagens ou potências diferentes dos produtos inovadores.

b. Produto Farmacêutico Inovador - é aquele inicialmente licenciado para comercia-lização, como medicamento patenteado, com base nas informações de segurança, qualidade e eficácia exigidas pela legislação.

c. Produto Farmacêutico de Referência geralmente é um produto farmacêutico colocado no mercado, com o qual um novo produto pretende ser intercambiável na prática clínica. Tanto pode ser o produto inovador ou o produto líder de mercado.

d. Equivalência Farmacêutica - diferentes produtos são equivalentes farmacêuticos se contêm a mesma quantidade da mesma substância(s) ativa(s), na mesma dosagem, de acordo com os mesmos padrões e para ser administrados pela mesma via. A equivalência farmacêutica não necessariamente implica em bioequivalência, posto que diferenças nos excipientes ou no processo da produção podem levar a diferenças no desempenho do produto, no que tange à dissolução e/ou biodisponibilidade.

e. Biodisponibilidade - consiste na velocidade e extensão da absorção de um princípio ativo de uma determinada forma farmacêutica, como determinado pela sua curva de concentração/tempo na circulação sistêmica ou pela sua excreção na urina.

f. Bioequivalência - dois medicamentos são bioequivalentes se eles são farmaceuticamente equivalentes e suas biodisponibilidades, após 
administração na mesma dose molar, são similares a tal grau que seus efeitos, com respeito à eficácia e segurança, sejam essencialmente os mesmos.

Cabe às autoridades regulatórias nacionais assegurar a execução das análises necessárias para garantir a qualidade e eficácia dos produtos circulando no mercado. A OMS recomenda a padronização de estudos de equivalência de medicamentos, com a finalidade de que sejam considerados intercambiáveis. Nesse sentido, as análises principais são a biodisponibilidade comparativa (bioequivalência), estudos fármacodinâmicos comparativos em humanos, testes clínicos comparativos e testes in vitro, principalmente dissolução.

Em que pese a base legal no Brasil, que permite a normatização de ensaios e testes, cabe lembrar que temos tido uma vigilância sanitária inexpressiva, pouco atuante e desaparelhada. A legislação brasileira assegurou o direito ao registro de produtos similares, mas não regulamentou as exigências necessárias no que se refere a bioequivalência. Complementarmente à Lei 6.360/76 e ao Decreto 79.094/77, a então Câmara Técnica de Medicamentos baixou a Resolução 04/78, considerando como medicamentos similares aqueles que contêm as mesmas substâncias ativas e que possuem indicação e posologia semelhantes a medicamentos anteriormente registrados para uso no país. Em nenhum momento, esta resolução exigia a comprovação de testes referentes a dissolução, biodisponibilidade ou bioequivalência. Fica claro que no Brasil a atual legislação define o que são produtos farmaceuticamente equivalentes, mas não regulamenta a bioequivalência.

\section{A POLÍTICA DA OMS}

A Organização Mundial da Saúde (OMS), desde a implantação do Programa de Ação de Medicamentos Essenciais em 1981 (Fefer, 1993), vem colaborando com os países na formulação de políticas e programas para assegurar a disponibilidade de medicamentos essenciais. Nos seus documentos mais recentes, a questão dos medicamentos genéricos vem sendo cada vez mais enfatizada, no sentido de representar uma alternativa para assegurar a disponibilidade de medicamentos a preços mais baixos.

Um dos marcos mais recentes e importantes nesse sentido foi a Conferência Latino-americana sobre aspectos econômicos e financeiros dos medicamentos essenciais, realizada em Caracas, Venezuela, em março de 1992, durante a qual foi reiterada a política de medicamentos essenciais como um componente básico da política de saúde, ao mesmo tempo atribuindo uma maior importância aos mecanismos de mercado. $\mathrm{O}$ ponto central da estratégia de uma política de medicamentos essenciais no atual contexto é a adoção de programas de medicamentos genéricos, entendendo assim a comercialização de produtos rotulados exclusivamente de acordo com a DCI e com características de intercambialidade com os produtos de marca (OMS, 1992). A análise procedida durante a referida Conferência é de que a adoção de programas de medicamentos genéricos nos países da América Latina viria otimizar o mercado, ao romper com a exclusividade das marcas comerciais, oferecendo alternativas à população, reduzindo os preços e racionalizando os gastos dos sistemas públicos de compra e abastecimento.

A $46^{\mathrm{a}}$ Assembléia Mundial da Saúde, ocorrida em 1993, discutiu especificamente a recomendação aos países membros de adotarem a denominação genérica dos medicamentos em destaque nos rótulos e propagandas de produtos farmacêuticos, por intermédio da legislação e regulação correspondentes (WHO, 1993a).

\section{A SITUACÃO MUNDIAL: EXEMPLOS E EVOLUÇÃO}

Consideramos oportuno fazer algumas considerações sobre a situação mundial, no que tange ao estado atual de mercado para os medicamentos genéricos e as características gerais das políticas que vêm sendo implementadas.

Nos Estados Unidos, a partir de 1984, com a promulgação do "Drug Price Competition and Patent Term Restoration Act", conhecido como Waxman - Hatch Act, ao mesmo tempo em que se estendeu a proteção patentária às empresas inovadoras, as empresas que optam por lançar produtos genéricos tiveram seu procedimento de registro simplificado no que se refere aos testes 
clínicos de eficácia e segurança (Seife, 1990). Entretanto, cabe ressaltar que na documentação constante da ANDA (Abbreviated New Drug Application) é obrigatório mencionar os protocolos e estudos de biodisponibilidade, bioequivalência e dissolução in vitro.

A evolução do mercado de medicamentos genéricos em vários países pode ser apreciado na Tabela 3. $\mathrm{O}$ aumento do mercado de genéricos nos EUA foi acompanhada de um acréscimo em outros países. Outros dados que merecem ser citados são referentes à Dinamarca, onde os genéricos respondem por $50 \%$ do mercado varejista e os Países Baixos, onde representam $40 \%$. Bélgica, Portugal e Espanha são exemplos de países onde os genéricos ainda representam mercados incipientes (Gerez, 1993a).

A Argentina, ou mais especificamente a Província de Buenos Aires, formulou seu Programa de Medicamentos, contemplando uma série de objetivos específicos, a partir da constatação de que, em 1985, 30\% dos gastos do total de assistência à saúde se referiam ao consumo de medicamentos. O Programa de Medicamentos foi sendo consolidado através de uma série de atos governamentais seqüenciais e complementares, incluindo decretos, resoluções e projeto de lei (Garcia, 1993).

O Canadá estabeleceu em 1969 a Lei do Licenciamento Compulsório, que permitia que houvesse a produção de medicamentos sob patente, com o pagamento de royalties ( $4 \%$ das vendas) à empresa inovadora. Hoje se estabelece um preço mínimo dos produtos intercambiáveis para o reembolso dos medicamentos. São exigidas provas de bioequivalência e são divulgadas listas dos produtos intercambiáveis. $\mathrm{O}$ farmacêutico pode substituir entre os produtos listados e o médico tem o direito de mencionar "não substituível” em suas prescrições (Kay, 1993).

TABELA 3. Percentuais de Mercado de Genéricos 1980 a $1985^{\star}, 1993^{\star \star}$

\begin{tabular}{lccccccc}
\hline \hline País & 1980 & 1981 & 1982 & 1983 & 1984 & 1985 & 1993 \\
\hline França & - & 1,0 & 1,0 & 2,0 & 2,0 & 3,0 & 13,0 \\
RFA & 1,5 & 1,5 & 2,5 & 3,0 & 4,0 & 5,0 & 35,0 \\
Inglaterra & 3,0 & 3,0 & 4,0 & 6,0 & 7,0 & 9,0 & 50,0 \\
Itália & 6,0 & 9,0 & 9,0 & 9,5 & 10,0 & 11,0 & - \\
Japão & 12,0 & 14,0 & 15,0 & 15,0 & 17,0 & 19,0 & - \\
Canadá & - & 14,1 & 17,5 & 18,5 & 19,5 & 21,3 & - \\
EUA & 21,0 & 21,0 & 22,0 & 22,0 & 24,0 & 25,0 & 30,0 \\
\hline \hline
\end{tabular}

* WHO - The World Drug Situation, 1988 (Scrip, 1987).

${ }^{* *}$ Gerez, J. C. C., 1993a.

A Colômbia promulgou o decreto 709 em 1990, estabelecendo agilização no registro de medicamentos, isenção de taxa, possibilidade de produzir genéricos com o mesmo registro de marcas e estabeleceu a obrigatoriedade de mencionar a denominação genérica nas embalagens no mesmo tamanho das marcas, além da prescrição pelo nome genérico. Houve ações judiciais contra o governo por parte das empresas multinacionais, reclamando da obrigatoriedade nas embalagens e contra a liberdade de prescrição. O Conselho de Estado deu despacho em fevereiro de 1993, mantendo o decreto, fortalecendo politicamente a ação governamental (Arango-Filho, 1993).

A Índia promulgou sua Política de Medicamentos em 1978, tendo a partir dessa data, praticamente eliminado a importação de produto final, fortalecendo sua indústria. Em 1986, foram introduzidas modificações, estabelecendo um sistema de preços, mudanças no registro de produtos e prioridade à produção de medicamentos essenciais. No que se refere a medicamentos genéricos, se discutiu a importância da montagem de uma estrutura sólida de laboratório para a realização de testes 
de bioequivalência e biodisponibilidade, com ênfase na Farmacologia Clínica (Sen \& Das Gupta, 1990).

\section{UMA ALTERNATIVA PARA O MERCADO BRASILEIRO}

É nítido que a produção de medicamentos genéricos representa uma tendência que se observa tanto em países desenvolvidos, como também a OMS recomenda sua implementação como espinha dorsal de uma política para os países em desenvolvimento. Acreditamos que, para o mercado brasileiro, também os medicamentos genéricos representam uma alternativa concreta, desde que uma série de conceitos e procedimentos sejam definidos e implementados pelos diferentes atores envolvidos no processo.

A simples existência de produtos com denominação genérica, não é suficiente para estabelecer a competitividade necessária ou influenciar os preços de mercado. É necessário o estabelecimento de uma política governamental clara, incluindo mecanismos de pactuação com a indústria, compro-metimento das diversas categorias de profissionais envolvidos, apoio da sociedade científica e entidades de saúde e todo o arcabouço jurídico complementar necessário.

É necessário esclarecer uma série de conceitos, no que se refere a medicamentos genéricos e o mercado brasileiro. As indústrias multinacionais no Brasil fazem críticas e restrições ao decreto dos genéricos, alegando que a substituição ou a prescrição de medicamentos genéricos vai acarretar problemas com a qualidade dos medicamentos. Essa crítica se aplica aos registros por similaridade. Entretanto, é claro que a questão de monitorar e assegurar a qualidade dos produtos circulando no mercado é um ponto crucial na implementação de uma política de medicamentos genéricos e deve ser assumida como ação governamental.

Outra questão a esclarecer é diferenciar "medicamentos genéricos" e a denominação genérica de produtos já existentes no mercado. Nesse sentido, vimos as conceituações no que tange a intercambialidade, bioequivalência e biodisponibilidade.

\section{O PROGRAMA DE ASSISTÊNCIA FARMACÊUTICA E ODECRETO 793: AVANÇOS E ENTRAVES}

Em decorrência das condições políticas na época, em acordo com recomendações da OMS e com base na experiência de outros países, o decreto 793, publicado no Diário Oficial da União de 6/4/93 e inserido no âmbito do Programa de Assistência Farmacêutica elaborado pelo Ministério da Saúde (MS, 1993), apresenta os seguintes avanços no que se relaciona à política de medicamentos no país:

1. determina o destaque à denominação genérica dos medicamentos em relação aos nomes de marca;

2. determina também a prescrição pela denominação genérica, ao mesmo tempo em que não proíbe a utilização das marcas de fantasia;

3. obriga a presença do farmacêutico nas farmácias, o que vem apenas reiterar legislação já existente no Brasil, além de ser prática rotineira na maioria dos países;

4. permite o fracionamento das embalagens de medicamentos, desde que garantidas a qualidade e eficácia terapêutica originais.

Uma série de entraves vem dificultando a efetiva implementação do decreto, cabendo destaque às numerosas ações na Justiça, as pressões exercidas por representações diplomáticas, as fragilidades do atual sistema nacional de vigilância sanitária e a não-concretização de ações a cargo da esfera federal, como é o caso da divulgação ampla da correlação entre as denominações genéricas e os nomes de marca.

\section{PERSPECTIVAS E PROPOSTAS: AÇÕES NECESSÁRIAS}

A implementação efetiva de uma política de medicamentos genéricos deve ser encarada como uma alternativa para o mercado brasileiro, considerando que a experiência de outros países mostrou que efetivamente reduz os preços, ao romper com o monopólio das marcas e propiciar opções à população. Entretanto, é necessária uma série de ações governamentais para 
assegurar a qualidade e equivalência dos produtos licenciados. As dificuldades na implementação do decreto 793/93 mostraram que há conflitos entre os interesses sociais de políticas de saúde e interesses comerciais de empresas farmacêuticas. Ao mesmo tempo em que se pactuam mecanismos de relação, o poder regulador do Estado deve ser exercido em sua plenitude e dentro das margens que nossa legislação estabelece.

Uma proposta de política de medicamentos genéricos tem que se constituir em uma ação setorial, envolvendo outros ministérios e outras esferas de governo, além da sociedade. Desde que ela seja assumida com respaldo político governamental, os pontos a seguir representam questões que precisam ser equacionadas de maneira integrada para implantar um programa deste tipo:

1. Revisão no registro dos medicamentos licenciados, diferenciando aqueles que se encontram efetivamente circulando no mercado.

2. Análise dos produtos registrados por grupos terapêuticos, com identificação do produto inovador e suas respectivas análises fármaco dinâmicas.

3. Estabelecimento dos requisitos de bioequivalência e biodisponibilidade, com o elenco de provas a realizar e suas equivalências in vitro, a ser determinados por Portaria Ministerial.

4. Montagem e certificação da infra-estrutura no Instituto Nacional de Controle de Qualidade em Saúde (INCQS/Fiocruz), capaz de assegurar o respaldo técnico e capacidade de análise necessários.

5. Desencadeamento imediato de programas regulares de inspeção a indústrias, com ênfase em Boas Práticas de Produção (GMP) e na verificação dos laboratórios de controle da qualidade.

6. Estabelecimento de políticas de preços de referência de medicamentos e cadastro de empresas candidatas à produção de medica mentos genéricos, com mecanismos claros de articulação governo - empresa.
7. Ações complementares, incluindo a efetiva implementação do Decreto 793, fortalecimento dos laboratórios estatais, estabelecimento de laboratórios de controle da qualidade auxiliares, além do arcabouço jurídico e instrumental legal necessários.

\section{RESUMO}

BERMUDEZ, J. Medicamentos Genéricos: Uma Alternativa para o Mercado Brasileiro. Cad. Saúde Públ., Rio de Janeiro, 10 (3): 368-378, jul/set, 1994.

A análise das características da indústria farmacêutica no mundo e os aspectos mercadológicos desse segmento no Brasil deixam evidentes a dependência externa e a oligopolização, com predomínio de empresas de caráter mutinacional, refletindo uma tendência mundial.

As iniciativas governamentais recentes no Brasil, com o objetivo de diminuir essa dependência, aliadas à gravidade da questão dos preços e custos de medicamentos no Brasil, comparados a preços mundiais, fundamentam a proposta de avaliar os medicamentos genéricos como uma alternativa viável para o mercado brasileiro. Um dos aspectos mais alarmantes é a verificação do superfaturamento de matériasprimas evidenciada pelos denominados "preços de transferência”, prática constatada em diversos países.

Analisando os conceitos internacionais sobre medicamentos genéricos, denominação genérica e equivalência farmacêutica, com base ainda em recomendações da Organização Mundial da Saúde e avaliando as experiências de diversos países, são propostas uma série de medidas destinadas a implementar, gradativamente, uma política de medicamentos genéricos bioequivalentes e intercambiáveis como um dos mecanismos de regulação do mercado brasileiro.

Palavras-Chave: Medicamentos Genéricos; Política de Medicamentos e Saúde; Indústria Farmacêutica; Brasil: Mercado de Medicamentos 


\section{REFERÊNCIAS BIBLIOGRÁFICAS}

ABBAS, R. \& BERMUDEZ, J., 1993. Preços no Brasil superam em muito os do mercado internacional. TEMA/RADIS, 11: 10-12.

ABIFARMA (Associação Brasileira da Indústria Farmacêutica), 1987. A Indústria Farmacêutica no Brasil: A Realidade. Rio de Janeiro: Pacheco.

ABIFINA (Associação Brasileira das Indústrias de Química Fina); ABQ (Associação Brasileira de Química) \& ABEQ (Associação Brasileira de Engenharia Química), 1992. Indústrias de Química Fina no Brasil. Situação Atual e Perspectivas para sua Integração na Economia Mundial. Petrópolis: Serrana.

ALANAC (Associação dos Laboratórios Farmacêuticos Nacionais), 1989. Sugestões sobre o Setor Industrial Farmacêutico. São Paulo: Alanac. (Mimeo.)

ALIFAR (Asociación Latinoamericana de Industrias Farmacéuticas), 1988. Industria farmacéutica latinoamericana. Industria Nacionaly Seguridad Latinoamericana, Buenos Aires, 7: 06-10.

ARANGO-FILHO, J. I., 1993. Comentários para una Propuesta de Medicamentos Genéricos. Seminário Internacional sobre Medicamentos Genéricos. Brasília: MS/OMS/ OPS. (Mimeo.)

BANCO MUNDIAL, 1993. Relatório sobre o Desenvolvimento Mundial 1993. Investindo em Saúde. Rio de Janeiro: Fundação Getúlio Vargas.

BERMUDEZ, J., 1992. Remédios: Saúde ou Indústria? A Produção de Medicamentos no Brasil. Rio de Janeiro: Relume Dumará. 1994. Acesso e Qualidade; Qual é o modelo viável? Análise crítica da política de medicamentos no Brasil. Rio de Janeiro: Escola Nacional de Saúde Pública. (Mimeo.) CEME (Central de Medicamentos), 1987a. Medicamentos Essenciais. Medidas para Assegurar o Abastecimento Interno. Brasília: Minitério da Saúde.

1987b. Medicamentos Essenciais.

Os Caminhos da Autonomia. DocumentoProposta. Brasília: Ministério da Saúde.

CODETEC (Companhia de Desenvolvimento Tecnológico), 1991a. A Indústria Farmacêutica. Diagnóstico e Perspectivas. Campinas: Codetec. (Mimeo.) 1991b. Patentes Farmacêuticas. Campinas: Núcleo Setorial de Informação em Química Fina/SCT/PR/CNPq/IBICT/ Codetec. (Mimeo.) 1991c. Apresentação da Instituição. Campinas: Codetec. (Mimeo.) COLÔMBIA (Ministerio de la Salud), 1992. Los Medicamentos en Colombia. Sólo Algunos son Esenciales. Bogotá: Trazo Ltda.

FEFER, E., 1993. El programa regional de medicamentos esenciales de la Organización Panamericana de la Salud y Organización Mundial de la Salud. Boletin Informativo de Medicamentos, Bolívia, 8: 2-3.(Número Especial)

FRENKEL, J.; REIS, J. N.; ARAÚJO Jr., J. T.; NAIDIN, L. C.; LOBÃO, R. S. \& FONSECA, M. S., 1978. Tecnologia e Competição na Indústria Farmacêutica Brasileira. Rio de Janeiro: Finep. (Mimeo.)

GARCIA, G. G., 1993. Programa de Medicamentos de la Província de Buenos Aires. Seminário Internacional sobre Medicamentos Genéricos. Brasília: MS/OMS/OPS. (Mimeo.)

GEREZ, J.C.C., 1993a. A Indústria Farmacêutica no Brasil e a Perspectiva para uma Política Nacional de Medicamentos Genéricos. Seminário Internacional sobre Medicamentos Genéricos. Brasília: MS/OMS/OPS. (Mimeo.) 1993b. Indústria Farmacêutica: histórico, mercado e competição. Ciência Hoje, 15: 21-30.

KAY, J. M.,1993. Generic Dugs: the Canadian Experience. Seminário Internacional sobre Medicamentos Genéricos. Brasília: MS/ OMS/OPS. (Mimeo.)

KOROLKOVAS, A., s/d. Denominações Comuns de Fármacos. São Paulo: Faculdade de Ciências Farmacêuticas/USP. (mimeo.)

MARTINEZ, M.N. \& GONÇALVES,O. R., 1990. Pesquisa \& Desenvolvimento, no Campo dos Insumos para a Saúde - Tecnologias e Patentes. Brasília: MS. (Mimeo.)

MS (Ministério da Saúde), 1993. Programa de Assistência Farmacêutica. Brasília: MS. (Mimeo.)

OMS (Organización Mundial de la Salud), 1992. Conferencia Latinoamericana sobre Aspectos Económicos y Financieros de los Medicamentos Esenciales. Informe. Caracas: OMS/Opas. (Mimeo.) 
PALÁCIOS, T., 1962. A Indústria Farmacêutica em País Subdesenvolvido. Anais do XV Congresso Brasileiro de Higiene. Recife. (Mimeo.)

SCRIP, 1990. World Pharmaceutical News. Review Issue 1990. London: PJB Publications Ltd.

SEIFE, M.,1990. Evolution of the principle US food and drug laws. In: Generic Drugs, Bioequivalence and Pharmacocinetics (K. N. Sharma, K. K. Sharma \& P. Sen, eds.), pp. 7-14, Delhi: Nirmal Vijay Printers.

SEN, P. \& DAS GUPTA, P., 1990. Bioequivalence of generic drugs: current status, and problems in the indian context. In: Generic Drugs, Bioequivalence and Pharmacocinetics (K. N. Sharma, K. K. Sharma \& P. Sen, eds.), pp. 91-94, Delhi: Nirmal Vijay Printers.
WHO(World Health Organization), 1993a. Forty Sixth World Health Assembly. International Nonproprietary Names for Pharmaceutical Prepaations. Report by the Director-General. Geneva: WHO, Provisional Agenda. (Mimeo.)

1993b. Interchangeable Multi-Source Pharmaceutical Products. WHO draft guideline on marketing authorization requirements. Geneva: WHO, Consultative Document. (Mimeo.) 